\title{
Effects of long term high cholesterol diet on spatial learning in aged rats
}

\author{
Shuang Rong\#, Rui Xu\#, Jing Cheng, Meng Chen, Chengcheng Ding, Jing Zeng* and Wenfang Li* \\ Department of Public Health School, Wuhan University of Science and Technology, Wuhan, 430065, China \\ "Contributed equally to this study
}

\begin{abstract}
Aims: The present study aimed to determine the effect of a long-term cholesterol enriched diet on spatial learning performance in middle to old aged rats.

Materials and methods: Based on initial Morris water maze results, 40 female SD rats aged 12 -month were screened and randomly divided into 2 groups. Two groups of female SD rats were fed either a balanced diet or a high cholesterol diet for 6 months on their 12-month aged of life ( $\mathrm{n}=20$ per group). At the end of the dietary intervention, behavioral testing by the Morris water maze task was applied again at 18 months to evaluate the spatial learning of aged rats by measuring latency, compared to controls.

Results: Consumption of a long term high cholesterol diet for 6 months significantly elevated serum cholesterol levels reported to controls. During the transition period from middle age to old age, rats fed with cholesterol enriched diet exhibited a longer escape latency than that of control group.
\end{abstract}

Conclusion: Our findings provided evidence that a long-term cholesterol-treated rats impaired aged rats' spatial learning.

\section{Introduction}

The experimental studies have confirmed that high cholesterol diet is detrimental to cognitive performance in animal models but the findings were mostly performed in young adult animals [1]. Results have confirmed that consumption of a western diet $(21 \%$ fat, $0.15 \%$ cholesterol) or high fat diet $(60 \%$ fat) for twelve weeks impaired rat's spatial memory [2]. Diets rich in high cholesterol have been shown to be detrimental to the hippocampi of adult and aged rats [3]. Hypercholesterolemia in adult rats has shown an impaired learning and long-term memory, which resemble an Alzheimer's disease (AD)-like pathology [4]. Interestingly, an animal model research found that cognitive deficits of age-related may be protected by hypercholesterolemia [5], although the underlying cause remains unclear.

Alternatively, A dietary intervention study was performed that a saturated fat and high cholesterol diet profoundly impaired memory and hippocampus morphology of the middle-aged rat [6]. Van Vliet et al. reviewed that a high serum cholesterol level measured at midlife associates with an increased risk of late-life cognitive decline, but the association is strongly age-dependent [7]. Therefore, early intervention focused on cholesterol management is likely to have a benefit on cognitive decline [8]. Since fewer studies were performed in this aspect, and the long-term effect of such diets on cognitive function in older animals is yet unknown. Current limitations in terms of this dietary intervention are performing at several decades from middle age to old age in the study of population are required as well. The main challenge of studying cognitive decline is the duration to observe such an effect from middle age to old age. Therefore, we fed a cholesterol enriched diet to 12 -month aged rats for 6 months, which simulate the natural aging process of human being from midlife to older [9-12]. The purpose of our present study is designed to assess the effects of long-term high cholesterol diet intervention on spatial learning performance of middle-aged rats by using the Morris water maze.

\section{Materials and methods}

\section{Animals}

Eighty 8-month-old female Sprague-Dawley rats (weighted 336.95 $\pm 42.00 \mathrm{~g}$ ), were obtained from Hubei province center of disease control and prevention (Wuhan, China). Five rats were housed per cage and maintained at a controlled ambient temperature $\left(24 \pm 1^{\circ} \mathrm{C}\right)$ under diurnal conditions (light-dark: 08:00-20:00) with access to laboratory chow and tap water ad libitum. Adequate levels of essential nutrients were provided daily. The health and dietary intake of the animals was monitored weekly, and any animals with overt signs of chronic respiratory distress, infection, or tumors were removed from this study. The animals were weighed every week during the experiment. The animals were cared for in accordance with the Guiding Principles in the Care and Use of Animals (2011). All animal experiments were approved by the Medical College Council on Animal Care Committee, Wuhan University of Scienc and Technology, China.

Correspondence to: Jing Zeng, Department of Public Health School, Wuhan University of Science and Technology, Wuhan, 430065, China, Tel: 13797020322; E-mail: zengjingwhu@163.com

Wenfang Li, Department of Public Health School, Wuhan University of Science and Technology, Wuhan, 430065, China, Tel: 13871358879; E-mail: Li-WF@163.com

Key words: aged, long term, cholesterol, spatial learning, morris water maze

Received: November 13, 2016; Accepted: December 11, 2016; Published: December 14, 2016 


\section{The Morris water maze}

Water maze testing was performed by the method of Morris (1984), which is commonly used test of spatial learning and memory in rodents and all experiments were carried out between 10:00 and 15:00 [13]. The Morris water maze (SuperMaze, XR-XM101) is a circular pool (160 $\mathrm{cm}$ in diameter and $50 \mathrm{~cm}$ in height) which contains no internal cues, stimuli, markings, or objects, but is surrounded by stable, salient extramaze cues. The pool was filled to water at a temperature of $22 \pm 1^{\circ} \mathrm{C}$ and made opaque with $20 \mathrm{ml}$ black nontoxic carbon ink. The pool was conceptually divided into four quadrants of equal area (NE, SE, SW or $\mathrm{NW})$. The escape platform (12 cm in diameter and $15 \mathrm{~cm}$ in height) was submerged approximately $1 \mathrm{~cm}$ below the surface of the water and placed in the center of the SW quadrant, and it was located in the same position on every trial, whose top lies approximately $1 \mathrm{~cm}$ beneath the water surface. On the day before the experiment, the rats were placed in the pool and allowed to swim for $60 \mathrm{~s}$ in the absence of the escape platform. A trial began by placing a rat in the water with its head facing the wall of the pool at one of the three starting positions located in the NE, SE and NW quadrant, respectively. The sequence of the starting positions remained unchanged for all rat within one session, but was changed on each day. During the five subsequent experimental days, a test of four trial session is performed each day with an intertrial interval of $5 \mathrm{~min}$ in which the platform is not presented. Once the mouse found the platform, then it was allowed to remain on it for $10 \mathrm{sec}$. If the mouse failed to find the platform within $60 \mathrm{~s}$, the mouse was placed on the platform for $10 \mathrm{sec}$. The platform position, the experimental environment and the reference were unchanged throughout the experiment. Cognition ability was performed by searching for the same hidden platform in the target quadrant, which more experimental parameters including the escape latency, the total distance of swimming and the average swimming speed. During each trial session, escape latency (the time taken to find the platform) in $\mathrm{s}$, swimming distance (length of swim path to reach the platform) in $\mathrm{mm}$, and average swimming speed in $\mathrm{mm} / \mathrm{s}$ were recorded by the surveillance cameras and computer, and analyzed by SuperMaze V2.0 system software.

\section{Study design}

Four months after start of the experiment, the study commenced when the rats were 12 months of age. Based on the results from Morris water maze screening and biochemical assays, behavioral tasks were performed during the animal's light cycle. The cognitive status of those rats whose mean escape latencies on days $3-5$ differed by $<1.5$ standard deviations (SDs) were screened in the experiment to ensure sufficient sample size and reduce the error by the individual differences.

Based on the screening results, 40 rats were finally included in the study and randomly divided into 2 groups, with 20 rats in each group: Control and HCD group. Rats in the control group were fed with a balanced diet, and the experimental high-cholesterol diet contained $5 \%$ egg yolk powder, $10 \%$ lard oil, $2 \%$ cholesterol, $0.3 \%$ bile salts, $0.2 \%$ propylthiouracil, $82.5 \%$ basic feed [14-16]. The animals were kept on the high-cholesterol or control diets for 6-mounth. After 6 months of experimental study, cognitive performances of aged rats were assessed by retraining the animals in the Morris water maze as before.

\section{Determination of glucose and lipid parameters in serum}

After screening, animals had blood collected from the top of the tail. After 6-month, the rats were sacrificed and blood was collected by heart puncture for serum separation and biochemical assays. Lipid profiles such as total cholesterol (TC), triglyceride (TG), highdensity lipoprotein cholesterol (HDL-C) and low density lipoprotein cholesterol (LDL-C) levels in serum from both groups were examined using appropriate kits (ZhongSheng BeiKong Bio Inst, Beijing, China).

\section{Statistical analysis}

Data are expressed as the mean \pm SEM (standard error of the mean). All statistical analyses were performed using SPSS 17.0 statistical software (SPSS Inc. Chicago, IL). The data on the Morris water maze were analyzed by using repeated measures of variance (ANOVA) test with group as the between subject factor and day as the within subject factor [group (2) $\times$ day (5)]. Fisher's post hoc tests were used for pairwise comparisons if the overall differences were significant $(P<0.05)$ [17]. Body weight and biochemical profiles data of rats were using to compare between both groups with an unpaired t-test. Statistical significance was set at $\mathrm{P}<0.05$ for all tests.

\section{Results}

\section{Body weight and lipid parameters}

The control group had a mean food intake of $212 \pm 15$ grams per day and the HCD diet group had a weekly intake of $194 \pm 11$ grams per cage (calculated by cage with 5 rats/cage). Thus, energy intake does not appear to significantly difference after 6 month of treatment. Before the dietary intervention, there is no statistical difference on body weights and lipid parameters between two groups $(\mathrm{P}>0.05)$. Analysis of body weight over the 6 experimental months demonstrated that rats fed the HCD diet were of a significantly lower body weight compared to controls. After 6-mouth treatment, the HCD group exhibited significantly elevated total serum TC levels $(t=-5.02 ; p<0.01)$, as well as LDL-C $(t=-4.688 ; p<0.01)$, and HDL-C levels were significantly decreased $(t=3.664 ; p<0.01)$ as compared to the control group at the end of experiment. No significant difference was noted on the level of TG between two groups. The data suggested that the rat model with experimental hypercholesterolemia was successfully established (Table 1).

\section{Effect of 6-month cholesterol diet on the Morris water-maze learning performances.}

Before experiment, the escape latencies showed no significant differences between 2 groups during all the training days $\left(F_{(2,25)}=0.362\right.$, $P>0.05)$, as performed in Figure 1. After 6 months, the mean escape latency of the control group on training days was significantly shorter than that of before, which represented that there may exist spatial longterm learning in controls $(P<0.05)$. However, escape latency of high cholesterol group was significantly longer than that of before $(P<0.05)$

Table 1. Mean \pm SD of the body weight, serum lipid levels $(\mathrm{mmol} / \mathrm{L})$ of aged rats in experimental groups.

\begin{tabular}{|c|c|c|c|c|c|c|c|c|c|c|}
\hline \multirow[b]{2}{*}{ Groups } & \multicolumn{2}{|c|}{ Body weight (g) } & \multicolumn{2}{|c|}{ TC (mmol/L) } & \multicolumn{2}{|c|}{ TG $(\mathrm{mmol} / \mathrm{L})$} & \multicolumn{2}{|c|}{ LDL-C (mmol/L) } & \multicolumn{2}{|c|}{ HDL-C (mmol/L) } \\
\hline & Baseline & 6 months & Baseline & 6 months & Baseline & 6 months & Baseline & 6months & Baseline & 6 months \\
\hline Control & $334.6 \pm 52.2$ & $363.4 \pm 46.5$ & $1.44 \pm 0.3$ & $2.62 \pm 1.1$ & $1.08 \pm 0.6$ & $1.19 \pm 0.3$ & $0.18 \pm 0.07$ & $0.84 \pm 0.9$ & $1.20 \pm 0.2$ & $0.14 \pm 0.1$ \\
\hline HCD & $339.4 \pm 39.1$ & $274.5 \pm 37.4$ & $1.41 \pm 0.3$ & $6.88 \pm 3.1$ & $1.16 \pm 0.4$ & $1.08 \pm 0.1$ & $0.18 \pm 0.07$ & $4.90 \pm 3.2$ & $1.19 \pm 0.2$ & $0.07 \pm 0.03$ \\
\hline
\end{tabular}

TG: Triglyceride; HCD: High cholesterol diet group; LDL-C: Low density lipoprotein cholesterol; HDL-C: High density lipoprotein cholesterol; TC: Total cholesterol

Data was presented as Mean $\pm \mathrm{SD}$. 
(Table 2). As shown in Figure 2, long period cholesterol-treated rats exhibited a significant prolongation of mean escape latency compared with that of control group $(P<0.05)$ (Table 3$)$. A 2 (groups) $\times 5$ (days) analysis of escape latencies variance (ANOVA) with repeated measures of the factor revealed that all rats showed a progressive decline in escape latencies with training, and main effects for day $\left(F_{(4,28)}=46.31\right.$, $P<0.001)$ and for group $\left(F_{(1,28)}=5.296, P<0.05\right)$ were significant. Latencies in HCD group were significantly higher than controls on day 1 and day $4(P<0.01$, Fisher's post hoc test). These results indicate that the capacities of spatial learning of middle aged rats can be damaged by long time high cholesterol diet treatment.

There was no significant main effects on swimming distance and average swimming speed among groups for day $\left(F_{(2.02,25)}=42.21\right.$, $\left.P<0.001 ; \quad F_{(1.55,25)}=499.23, P<0.001\right)$ and for group $\left(F_{(1,25)}=0.894\right.$, $\left.P>0.05 ; F_{(1,25)}=4.075, P>0.05\right)$ (Figures 3 and 4 ). When compared with control group, HCD group at day 1 and day 5 showed a significant lower swimming speed $\left(F_{(1,25)}=6.25, P<0.01 ; F_{(2.02,25)}=42.21, P<0.01\right)$. Differences in escape latency caused by swimming ability and sensorimotor abilities of rats were excluded. Additionally, levels of

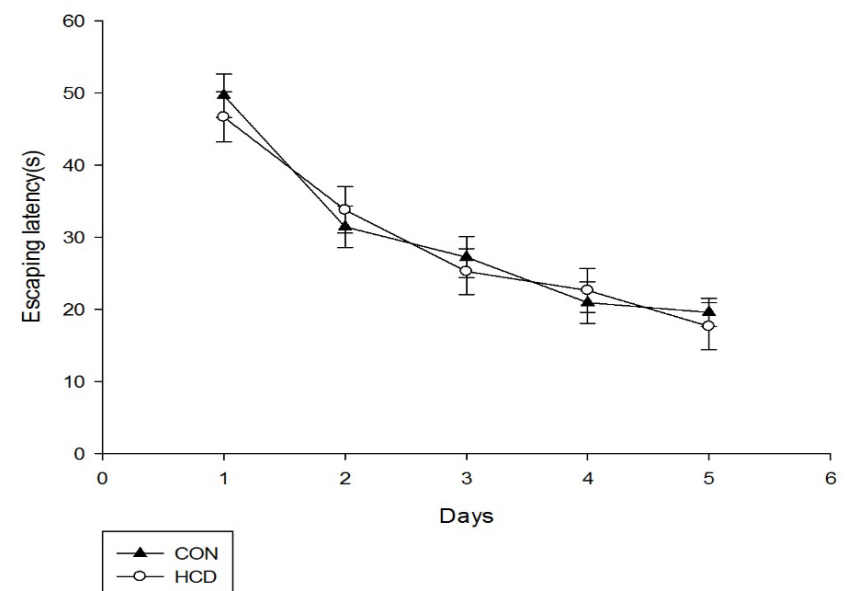

Figure 1. The spatial learning and memory performance of aged rats in Morris water maze during 5 days of testing in different groups at beginning of experiment [CON: The control group; HCD: High cholesterol diet. Each point represents the mean \pm SEM of escape latency].

Table 2. Paired comparison of average escaping latency of rats before and after the intervention.

\begin{tabular}{|c|c|c|c|c|c|}
\hline \multirow{2}{*}{ Groups } & \multicolumn{2}{|c|}{ Escaping latency (s) } & \multicolumn{3}{|c|}{ Paired t test } \\
\cline { 2 - 6 } & Before & After & $\bar{d}$ & $\mathrm{t}$ & $\mathrm{P}$ \\
\hline Control & $29.76 \pm 1.43$ & $22.56 \pm 1.29^{* *}$ & $-7.20 \pm 1.86$ & -3.86 & 0.001 \\
\hline HCD & $29.18 \pm 1.92$ & $34.07 \pm 2.21^{*}$ & $4.88 \pm 1.70$ & 2.88 & 0.016 \\
\hline
\end{tabular}

Control vs. HCD group, ${ }^{*} * \mathrm{P}<0.01, * \mathrm{P}<0.05$

Compared with the control group, there was a significant difference in HCD group by paired design $t$ test serum total cholesterol and escaping latency in aged rats were weakly correlated among all groups but this correlation was not observed with significant difference $(r=0.056, P=0.338)$.

\section{Discussions}

Several researches have shown that cholesterol levels elevated are considered as risk factors related to cognitive impairment [18]. Further, national clinical studies indicated high midlife serum total cholesterol was associated with poorer late-life cognition $[19,20]$. The use of statin, a group of commonly used cholesterol-lowering drugs, were reported to have the protective effect that early statin use was significantly associated with a reduction in $\mathrm{AD}$ progression in mild-tomoderate AD patients [21,22]. Hypercholesterolemia in mice turned out to induce short-term spatial memory impairments [23]. A diet rich in fat or cholesterol also demonstrated to have significant detrimental effect on cognitive performance and aging may make this effect more pronounced [24-27]. Therefore, the objective of this study was to evaluate the effect of long term cholesterol enriched diet on spatial learning performance of aged rats by the Morris water maze [17].

Considering the results of our study, the lower body weight found in HCD animals. Several studies found high fat diet-related impairment in behavioral tests with an associated increased in body weight. However, some interesting researches have reported the appearance of severe hypercholesteremic side effects that damage to the hippocampus can induce weight loss, appetite loss and disrupt the relationship between meal size and latency to start the next meal, which are consistent with our result $[28,29]$. Additionally, it is no doubt that a lower body weight may be partly due to a non-adaptation to this diet in HCD animals. Further, one study found that high fat diet exposure also resulted in

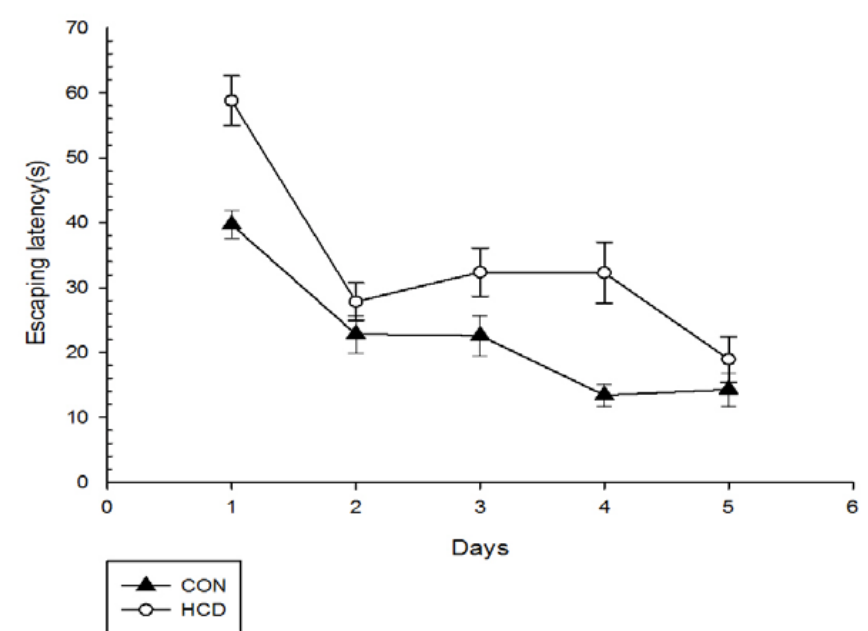

Figure 2. Effect of high cholesterol diet on escape latencies in Morris water maze of aged rats during 5 days of testing in aged rats [CON: Control group; HCD: High cholesterol diet group; Each point represents the mean \pm SEM]

Table 3. Comparison of average escaping latency, average total swimming distance and speed of rats before and after the intervention.

\begin{tabular}{|c|c|c|c|c|c|c|}
\hline \multirow{2}{*}{ Groups } & Escaping latency (s) & Total swimming distance (mm) & \multicolumn{2}{|l|}{ average swimming speed (mm/s) } \\
\cline { 2 - 6 } & Before & After & Before & After & $207.57 \pm 24.65$ \\
\hline Control & $29.76 \pm 1.43$ & $22.56 \pm 1.29$ & $9340.66 \pm 364.76$ & $3937.28 \pm 447.02$ & $216.07 \pm 51.23$ \\
\hline HCD & $29.18 \pm 1.92$ & $34.07 \pm 2.21^{* *}$ & $9128.91 \pm 389.46$ & $5123.09 \pm 511.19$ & $198.07 \pm 21.51$ \\
\hline $\boldsymbol{t}$ & 0.24 & -4.81 & 0.47 & 1.70 & 1.46 \\
\hline $\boldsymbol{P}$ & 0.808 & $<0.001$ & 0.643 & 0.103 & 0.150 \\
\hline
\end{tabular}

Control vs. HCD group, $* * \mathrm{P}<0.01$

Compared with the control group, there was a significant difference in HCD group by $\mathrm{t}$ test for completely randomized design 


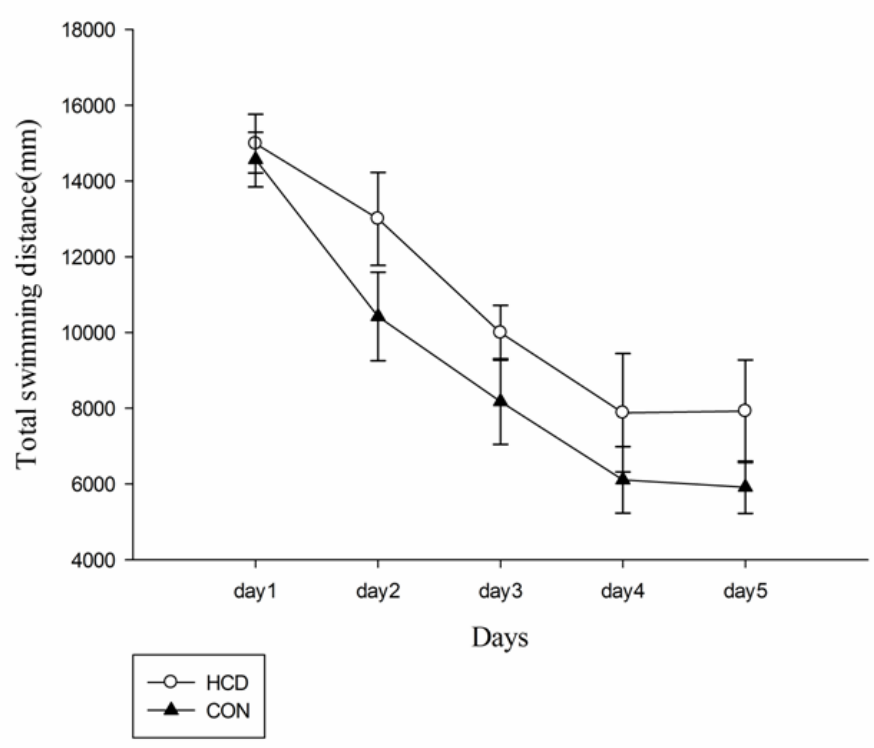

Figure 3. Effect of high cholesterol diet on total swimming distance in Morris water maze of aged rats during 5 days of testing in aged rats [CON: Control group; HCD: High cholesterol diet group; Each point represents the mean \pm SEM].

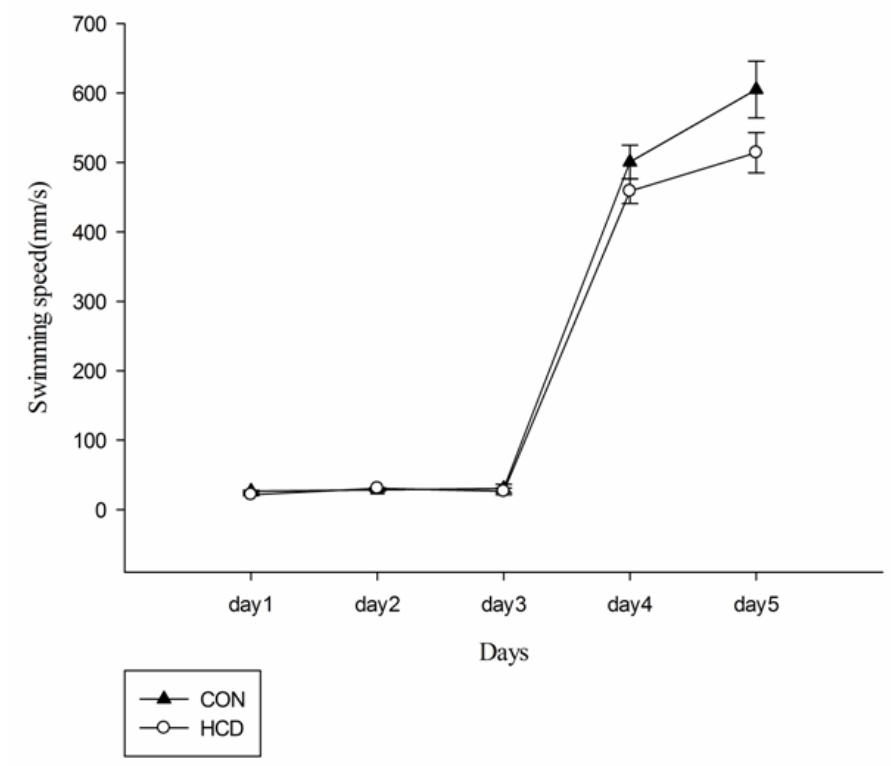

Figure 4. Effect of high cholesterol diet on the average swimming speed in Morris water maze of aged rats during 5 days of testing in aged rats [CON: Control group; HCD: High cholesterol diet group; Each point represents the mean \pm SEM].

deficits in the Morris Water Maze without affecting body weight [8]. In the present study, with the increased training days, the escape latency of rats in both groups showed a tendency of shortening. This phenomenon manifested that spatial learning performance of aged rats increased with the training times and spatial memory was discovered more memory retention [30]. Meanwhile, long period cholesterol diet exposure resulted in a significant increase in the escape latency compared to controls, implying that long-term dietary cholesterol consumption may impair spatial learning of aged rats. However, the correlation between serum TC and water-maze learning performances was not observed with middle aged rats for the reason that the levels of serum total cholesterol cannot represent the cholesterol metabolism in the brain.
Interestingly, a study by Dufour et al. [24] has revealed that onemonth-old male Wistar rats fed with a longer period of cholesterol enriched diet ( 2 or 4 months) performed a water-maze task more quickly than rats fed with a regular diet and tended to alter the retention of memory without affecting the improvement in the acquisition of the task $[24,25]$. Furthermore, they indicated that the detrimental effect of cholesterol on memory retention became evident only for older animals and depended on the duration of the diet. It seems to contradict with our results and previous studies, whose causes may be due to different types of animal models. Because we fed a longer period of cholesterol enriched diet to 12-month aged rats for the well-established model, which may only partially simulate natural ageing-related pathological changes of cognitive dysfunction. Additionally, long term high fat diet leading to (mild) ketonemia might result in better memory in elderly humans and in some animal models [31]. Therefore, the effect of high cholesterol diet on cognitive function still needs further exploration and research confirmed.

In conclusion, the present work suggests that a long term high cholesterol diet impairs seriously spatial learning deficits of aged rats. This discovery prompts us that early performed intervention of hypercholesterolemia is considered of strategic importance for improving the cognitive function in later life.

\section{Acknowledgements}

This work was supported by 2013 Newtrition"' Asia Research Grant by BASF. We acknowledge the contribution of Laboratory in Wuhan University of Science and Technology of Wuhan Province. We also thank all of the participants in this study for their contributions.

\section{Conflict of interest}

The authors declare to have no conflict of interest.

\section{References}

1. Ghribi O (2008) Potential mechanisms linking cholesterol to Alzheimer's diseaselike pathology in rabbit brain, hippocampal organotypic slices, and skeletal muscle. $J$ Alzheimers Dis 15: 673-684. [Crossref]

2. Kosari S, Badoer E, Nguyen JC, Killcross AS, Jenkins TA (2012) Effect of western and high fat diets on memory and cholinergic measures in the rat. Behav Brain Res 235: 98-103. [Crossref]

3. Abo El-Khair DM, El-Safti Fel-N, Nooh HZ, El-Mehi AE (2014) A comparative study on the effect of high cholesterol diet on the hippocampal CA1 area of adult and aged rats. Anat Cell Biol 47: 117-126. [Crossref]

4. Ullrich C, Pirchl M, Humpel C (2010) Hypercholesterolemia in rats impairs the cholinergic system and leads to memory deficits. Mol Cell Neurosci 45: 408-417. [Crossref]

5. Bohr I (2004) Hypercholesterolemic diet applied to rat dams protects their offspring against cognitive deficits. Simulated neonatal anoxia model. Physiology and Behavior 82: 703-711.

6. Granholm AC, Bimonte-Nelson HA, Moore AB, Nelson ME, Freeman LR, et al. (2008) Effects of a saturated fat and high cholesterol diet on memory and hippocampal morphology in the middle-aged rat. J Alzheimers Dis 14: 133-145. [Crossref]

7. van Vliet P (2012) Cholesterol and late-life cognitive decline. J Alzheimers Dis 30 Suppl 2: S147-162. [Crossref]

8. Anstey KJ, Lipnicki DM, Low LF (2008) Cholesterol as a risk factor for dementia and cognitive decline: a systematic review of prospective studies with meta-analysis. Am J Geriatr Psychiatry 16: 343-354. [Crossref]

9. Dutta S, Sengupta P2 (2016) Men and mice: Relating their ages. Life Sci 152: 244-248. [Crossref]

10. Sengupta P (2013) The Laboratory Rat: Relating Its Age with Human's. Int J Prev Med 4: 624-630. [Crossref] 
11. Flurkey KM, Currer J, Harrison DE (2007) Chapter 20 - Mouse Models in Aging Research A2 - Fox, James G. In: Davisson MT, Quimby FW, Barthold SW, Newcomer CE, Smith AL, editors. The Mouse in Biomedical Research (Second Edition). Burlington: Academic Press pp: 637-672.

12. National Research Council (2011) Committee for the Update of the Guide for the Care and Use of Laboratory Animals. Guide for the Care and Use of Laboratory Animals. 8th ed. Washington (DC): National Academies Press (US).

13. Xu J, Rong S, Xie B, Sun Z, Zhang L, et al. (2009) Procyanidins extracted from the lotus seedpod ameliorate scopolamine-induced memory impairment in mice. Phytotherapy Research 23: 1742-1747.

14. Madariaga YG, Cárdenas MB1, Irsula MT, Alfonso OC, Cáceres BA, et al. (2015) Assessment of four experimental models of hyperlipidemia. Lab Anim (NY) 44: 135140. [Crossref]

15. Kaizer RR, da Silva AC, Morsch VM, Corrêa MC, Schetinger MR (2004) Diet-induced changes in AChE activity after long-term exposure. Neurochem Res 29: 2251-2255. [Crossref]

16. Ando K, Higami Y, Tsuchiya T, Kanematsu T, Shimokawa I (2002) Impact of aging and life-long calorie restriction on expression of apoptosis-related genes in male F344 rat liver. Microsc Res Tech 59: 293-300. [Crossref]

17. Panza F, Solfrizzi V, D'Introno A, Colacicco AM, Santamato A, et al. (2009) Higher total cholesterol, cognitive decline, and dementia. Neurobiol Aging 30: 546-548. [Crossref]

18. Chou CY, Chou YC, Chou YJ, Yang YF, Huang N (2014) Statin use and inciden dementia: a nationwide cohort study of Taiwan. Int J Cardiol 173: 305-310. [Crossref]

19. Lin FC, Chuang YS, Hsieh HM, Lee TC, Chiu KF, et al. (2015) Early Statin Use and the Progression of Alzheimer Disease: A Total Population-Based Case-Control Study. Medicine (Baltimore) 94: e2143. [Crossref]

20. Moreira EL, de Oliveira J, Engel DF, Walz R, de Bem AF, et al. (2014) Hypercholesterolemia induces short-term spatial memory impairments in mice: upregulation of acetylcholinesterase activity as an early and causal event? J Neural Transm (Vienna) 121: 415-426.

21. Solomon A, Kivipelto M, Wolozin B, Zhou J, Whitmer RA (2009) Midlife serum cholesterol and increased risk of Alzheimer's and vascular dementia three decades later. Dement Geriatr Cogn Disord 28: 75-80. [Crossref]

22. Solomon A, Kåreholt I, Ngandu T, Winblad B, Nissinen A, et al. (2007) Serum cholesterol changes after midlife and late-life cognition: twenty-one-year follow-up study. Neurology 68: 751-756. [Crossref]

23. Dufour F, Liu QY, Gusev P, Alkon D, Atzori M (2006) Cholesterol-enriched diet affects spatial learning and synaptic function in hippocampal synapses. Brain Research 1103: 88-98.

24. van Vliet P, van de Water W, de Craen AJ, Westendorp RG (2009) The influence of age on the association between cholesterol and cognitive function. Experimental gerontology 44: 112-122.

25. Cordner ZA, Tamashiro KL (2015) Effects of high-fat diet exposure on learning and memory. Physiol Behav 152: 363-371. [Crossref]

26. Di Paolo G, Kim TW (2011) Linking lipids to Alzheimer's disease: cholesterol and beyond. Nat Rev Neurosci 12: 284-296. [Crossref]

27. D'Hooge R, De Deyn PP (2001) Applications of the Morris water maze in the study of learning and memory. Brain Res Brain Res Rev 36: 60-90. [Crossref]

28. Ghribi O, Larsen B, Schrag M, Herman MM (2006) High cholesterol content in neurons increases BACE, beta-amyloid, and phosphorylated tau levels in rabbit hippocampus. Experimental Neurology 200: 460-467.

29. Francis HM, Mirzaei M, Pardey MC, Haynes PA, Cornish JL (2013) Proteomic analysis of the dorsal and ventral hippocampus of rats maintained on a high fat and refined sugar diet. Proteomics 13: 3076-3091. [Crossref]

30. Solntseva SV, Storozheva ZI, Nikitin VP (2012) Long-term spatial memory retrieval at different times following formation in single session training in rats. Bulletin of Experimental Biology and Medicine 153: 617-619.

31. Setkowicz Z, Gazdzinska A, Osoba JJ, Karwowska K, Majka P, et al. (2015) Does Long-Term High Fat Diet Always Lead to Smaller Hippocampi Volumes, Metabolite Concentrations, and Worse Learning and Memory? A Magnetic Resonance and Behavioral Study in Wistar Rats. PloS One 10: e139987.

Copyright: @2016 Rong S. This is an open-access article distributed under the terms of the Creative Commons Attribution License, which permits unrestricted use, distribution, and reproduction in any medium, provided the original author and source are credited. 